\title{
ANÁLISE COMPARATIVA DE VIGAS BALDRAMES SOBRE APOIOS ELÁSTICOS
}

COMPARATIVE ANALYSIS OF FOUNDATION BEAMS ABOUT ELASTIC SUPPORTS

\author{
Renato Pedro Pereira da Silva' \\ Richard Ray Massa² \\ Kaliel Gomes Andrade ${ }^{3}$ \\ Tulio Raunyr Cândido Felipe ${ }^{4}$
}

\section{RESUMO}

O objetivo deste trabalho é avaliar os esforços solicitantes em vigas baldrame sem e com apoios elásticos e, comparar as suas respectivas taxas de armaduras à flexão. Para tal, são empregadas molas discretas independentes entre si com rigidez equivalente ao solo analisado. No tocante à rigidez longitudinal das molas usadas para a simulação, é utilizado o coeficiente de mola para solos argilosos. O dimensionamento dessas vigas é concretizado por meio das prescrições da ABNT NBR 6118:2014. Por fim, conclui-se que a utilização da abordagem proposta reduziu à taxa de armadura a flexão das vigas avaliadas em torno de 12,5\% para os momentos positivos e $18,7 \%$ para os momentos negativos.

Palavras-chave: Concreto armado. Interação solo estrutura. Vigas baldrame.

\section{ABSTRACT}

The goal of this work is to evaluate the efforts of foundation beams without and with elastic supports and to compare their respective rates of bending reinforcement. For this, discrete springs independent of each other are used with stiffness equivalent to the analyzed soil. As regards the longitudinal stiffness simulation, is used the spring coefficient for clayey soils. The design of these beams is effected through the requirements of ABNT NBR 6118:2014. Therefore, concluded the proposed approach reduced the rate bending reinforcement of the beams analyzed around $12,5 \%$ for positive bending moment and $18.7 \%$ for negative bending moment.

Keywords: Reinforced concrete. Soil-structure interaction. Foundation beams.

1 Graduando em Engenharia Civil pela Universidade Brasil. E-mail: renatopedro.eng@gmail.com 2 Graduando em Engenharia Civil pela Universidade Brasil. E-mail: richardmassa@hotmail.com.br 3 Graduando em Engenharia Civil pelo Centro Universitário de João Pessoa (UNIPÊ). E-mail: kaliel.gomes@hotmail.com 4 Doutorando em Engenharia de Estruturas pela Universidade de São Paulo (USP). Docente da Universidade Brasil. E-mail: tulioraunyr@usp.br 


\section{INTRODUÇÃO}

Toda a estrutura se deformará no ponto de conexão com o maciço de solo (CRESPO, 2004). Contudo, tradicionalmente os esforços nas estruturas são determinados presumindo apoios indeslocáveis, uma vez que nas hipóteses de cálculo o solo é assumido ser rígido (SANTOS, 2016a). Todavia, tais hipóteses não representam o comportamento físico do solo. Consequentemente, a interação entre o solo e a estrutura não é levada em consideração nos cálculos pertinentes.

Na literatura várias formulações para a implementação da interação soloestrutura (ISE) nos modelos de cálculos foram apresentadas ao longo dos anos. Dentre esses modelos o mais utilizado é o modelo de Winkler (SANTOS, 2016a; SANTOS, 2016b; TESTONI, 2013). O modelo Winkler (1867) é baseado no conceito de coeficiente de reação de uma mola uniaxial, a qual simula o solo por molas discretas independentes entre si. Além disso, o solo é assumido com comportamento elástico-linear. Adotando a hipótese que o solo é homogêneo e isotrópico, tal modelo pode ser empregado para ações verticais e horizontais (ANTONIAZZI, 2011). As vantagens da aplicação desse modelo são: redistribuições de esforços nos elementos estruturais e diminuição dos recalques diferenciais estimados pelo método de cálculo tradicional que assume apoios indeslocáveis (SANTOS, 2016a; TESTONI, 2013). Por outro lado, uma desvantagem desse modelo é que ele não considera a influência das estruturas vizinhas. Logo, a interação entre edificações próximas não é avaliada.

Com relação a redistribuições de esforços, ocorre um alívio de carga nos pilares mais carregados e a sobrecarga nos pilares menos solicitados (SOARES, 2005). Reis (2000) depreendeu que essas redistribuições de esforços ocorrem mais pronunciadamente nos pavimentos inferiores de uma edificação. Dessa forma, os elementos estruturais mais próximos do solo sofrem mudanças significativas em seus esforços, pelo fato de estarem mais próximo à ligação com a fundação e o solo. Essas redistribuições não podem ser ignoradas se tem como alvo uma estrutura com maior conforto, durabilidade e segurança (CRESPO, 2004).

No que concerne os recalques diferenciais, a solidariedade entre os componentes proporciona um aumento na rigidez, implicando em recalques menores do que os mensurados via método de cálculo tradicional (GUSMÃO, 1994).

A comunicação entre os engenheiros estruturais e geotécnicoséfundamental para uma análise de ISE. É ressaltado também que na maioria das estruturas essa interação entre profissionais não acontece, a menos que seja uma grande obra, que necessite de cuidados especiais (SANTOS, 2016a; SOUZA e REIS, 2008). Ademais, a ISE é governada pela interação entre a superestrutura, infraestrutura e terreno de fundação, desse modo a sua análise e seu desempenho é definido pela interação desses meios (SANTOS, 2016b; GUSMÃO, 1994). Assim sendo, denomina-se ISE o mecanismo de influência mútua entre a superestrutura e o sistema de fundação 
(TESTONI, 2013). Hoje em dia, alguns softwares comerciais já são capazes de levar em conta essa interação.

Atualmente vários trabalhos têm endereçado o acoplamento do Método dos Elementos Finitos com o Método dos Elementos de Contorno para avaliar a ISE (CAVALCANTI, 2006; MESQUITA e CODA, 2001). Esses métodos apresentam boa convergência dos resultados quando confrontados com os métodos clássicos de ISE.

De acordo com Antoniazzi (2011), a ISE é de grande importância em um projeto. Ela ao empregar ISE em vigas chegou a constatar pontos em que seus momentos fletores atuantes inverteram totalmente seu sentido de atuação. Essa inversão de esforços gerou a necessidade de aumentar as taxas de armadura em determinados pontos ao longo dessas vigas. Portanto, considerar somente os esforços atuantes sem a ISE pode levar a uma situação imprevista para uma estrutura.

Testoni (2013) aferiu que levando em conta a ISE na análise estrutural de estruturas de parede de concreto moldados no local alterou significativamente o fluxo de tensões entre os elementos estruturais.

Lourinho (2014) constatou que os valores de momentos fletores atuantes em uma estrutura variam com relação à rigidez relativa entre o solo e a estrutura. Segundo o referido autor, quando maior for essa rigidez, menor será o momento fletor. Por isso, mais uma vez ressalta-se a importância do estudo da ISE, principalmente em solos argilosos, a qual gera uma maior mudança nos esforços atuantes para a estrutura (LOURINHO, 2014). Esse é o tipo de solo escolhido neste trabalho para realizar as análises pertinentes de ISE.

No Brasil a ISE não é empregada pela maioria dos escritores de projetos (SANTOS, 2016a). Além disso, nas normas técnicas brasileiras encontram-se recomendações escassas com relação à consideração da ISE nos projetos estruturais. O item 14.2.2 da ABNT NBR 6118:2014 prescreve que em casos mais complexos a ISE deve ser contemplada na análise estrutural de edifícios de concreto armado. Entretanto, a referida norma não específica quais seriam esses casos complexos. $O$ item 11.5 da ABNT NBR 16055:2012 indica que o modelo com ISE é obrigatório para os casos de fundação em níveis diferentes em edificação de parede de concreto moldado no local. Ademais, essa norma recomenda que para estruturas com mais de cinco pavimentos, deve-se no mínimo considerar o modelo de molas discretas independentes localizadas nos pontos de apoio das vigas de fundação. Enquanto as normas ABNT NBR 15812-1:2010 e ABNT NBR 15961-1:2011 são omissas quanto à necessidade de incorporação da ISE na análise de estruturas em alvenaria estrutural de bloco cerâmico e bloco de concreto, respectivamente.

A comparação entre o método de ISE e o de um solo indeslocável mostra que existem diferenças nos esforços impostos aos elementos estruturais. Portanto, este trabalho pretende contribuir por meio da avaliação crítica do método de cálculo 
tradicional que assume apoios indeslocáveis, confrontando-o com o modelo de molas discretas independentes.

\section{OBJETIVO}

O objetivo deste trabalho é avaliar os esforços solicitantes em vigas baldrame sem e com apoios elásticos e, comparar as suas respectivas taxas de armaduras à flexão.

\section{METODOLOGIA}

Em uma primeira hipótese, assumindo os apoios indeslocáveis, foram determinados os esforços solicitantes das vigas baldrame de um edifício modelo. Posteriormente, como uma segunda hipótese, os apoios foram substituídos por molas discretas independentes entre si com rigidez equivalente do solo analisado, simulando assim de forma simplificada a ISE. Essa rigidez foi obtida para o solo argiloso, uma vez que esse solo implica em uma maior alteração nos esforços solicitantes em comparação aos outros tipos de solos (LOURINHO, 2014). Em ambas as hipóteses o dimensionamento foi efetivado seguindo as prescrições da ABNT NBR 6118:2014. Ademais, os esforços solicitantes foram apurados por meio do programa educacional FTOOL.

\section{RESULTADOS E DISCUSSÕES}

Para as discussões que se segue é utilizada a planta de forma das vigas baldrame de uma estrutura hipotética, conforme ilustrada na Figura 1.

Figura 1: Planta de forma das vigas baldrame da estrutura em análise.

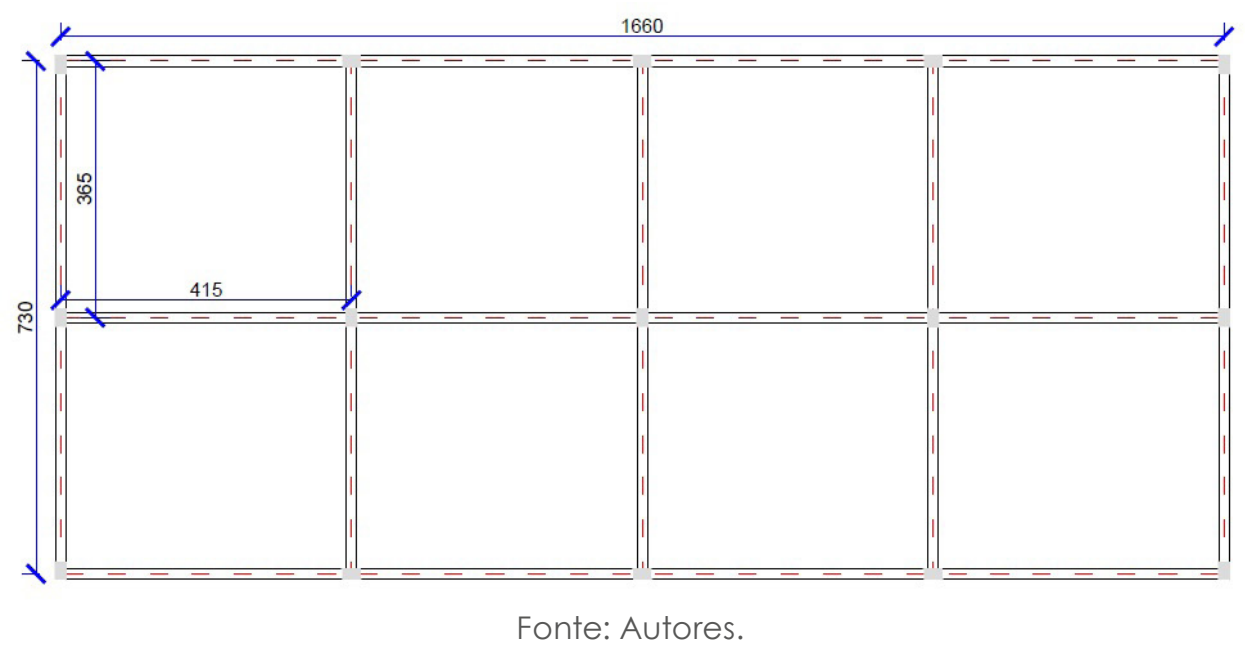


Na Figura 2 é exposta a numeração adotada para as vigas. Pelo prédimensionamento adotou-se que as vigas baldrame V01, V02 e V03 possuem 15 $\mathrm{cm}$ de espessura e $35 \mathrm{~cm}$ de altura. Já as vigas baldrame V04, V05, V06, V07 e V08 têm medidas de $15 \mathrm{~cm}$ de espessura e $30 \mathrm{~cm}$ de altura. Os valores anteriores foram determinados a partir da relação //12, sendo / o vão, conforme apresentado em Carvalho e Figueiredo (2014).

Figura 2: Numeração das vigas baldrame.

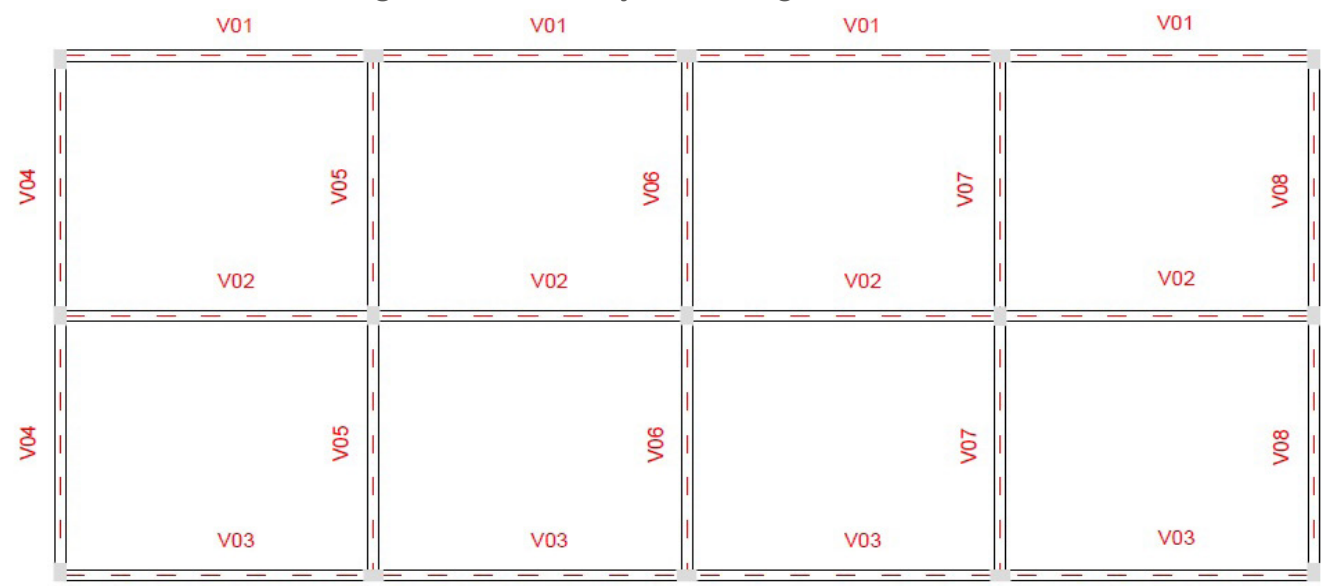

Fonte: Autores.

A Tabela 1 apresenta o peso específico dos materiais usados para determinar a carga utilizada para dimensionar as vigas baldrame, de acordo com a norma ABNT NBR 6120:1980.

Tabela 1: Peso específico aparente dos materiais.

\begin{tabular}{cc} 
Material & Peso especifico $\left(\mathrm{KN} / \mathrm{m}^{3}\right)$ \\
\hline Tijolo furado & 13 \\
\hline Argamassa de cal, cimento e areia & 19 \\
\hline Concreto armado & 25 \\
\hline
\end{tabular}

Fonte: ABNT NBR 6120:1980.

O módulo de elasticidade $(E)$ do concreto foi determinado de acordo com a norma ABNT NBR 6118:2014, considerando o agregado graúdo sendo granito e $f_{c k}$ igual a $25 \mathrm{MPa}$.

\section{ANÁLISE ASSUMINDO APOIOS INDESLOCÁVEIS}

Nesta seção são apresentados a análise estrutural e o dimensionamento admitindo a hipótese de apoios fixos para as vigas baldrame. Para determinação dos esforços solicitantes utilizou-se o programa educacional Fłool. Já com relação ao dimensionamento empregaram-se as prescrições da ABNT NBR 6118:2014. 
Conforme a ABNT NBR 6118:2014, para Combinação de Estado Limite Último (ELU), utiliza-se a seguinte equação:

$$
F_{d}=\sum \gamma_{g} \cdot F_{g k}+\gamma_{q} \cdot F_{q 1 k}+\sum \Psi_{0 j} \cdot F_{q j k} \cdot \gamma_{q}
$$

A Tabela 2 apresenta os valores da força de projeto $\left(F_{d}\right)$ aplicada a cada viga.

Tabela 2: Carga de projeto distribuída uniformemente ao longo da viga.

\begin{tabular}{cc} 
Viga & $\boldsymbol{F}_{d}(\mathrm{KN} / \mathrm{m})$ \\
\hline $\mathrm{V} 01=\mathrm{V} 02=\mathrm{V} 03($ seção $15 \times 35 \mathrm{~cm})$ & 11,54 \\
\hline $\mathrm{V} 04=\mathrm{V} 05=\mathrm{V} 06=\mathrm{V} 07=\mathrm{V} 08($ seção $15 \times 30 \mathrm{~cm})$ & 11,28 \\
\hline
\end{tabular}

Fonte: Autores.

Com os dados da Tabela 2, inserido-os ao Ftool, determinou-se os esforços solicitantes das vigas baldrame, em concordância com as Figuras 3 e 4.

Figura 3: Gráficos dos esforços solicitantes das vigas baldrame V01, V02, V03: a) carga de projeto; b) diagrama de força cortante; c) diagrama de momento fletor.

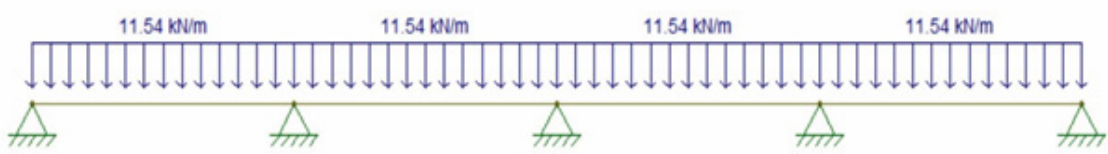

a)

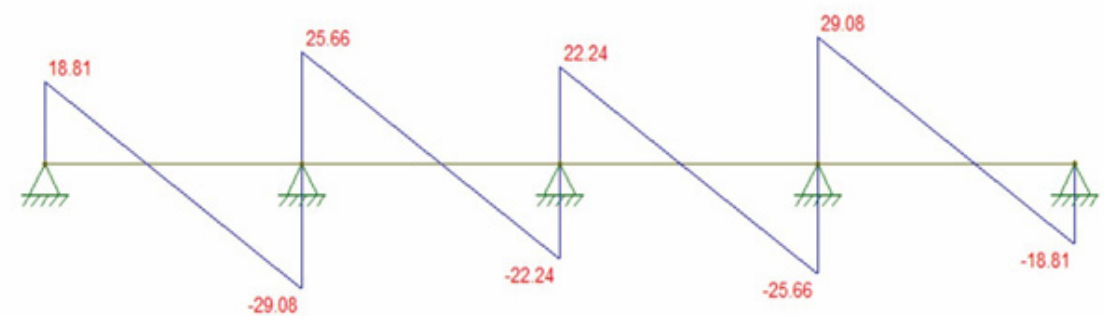

b)

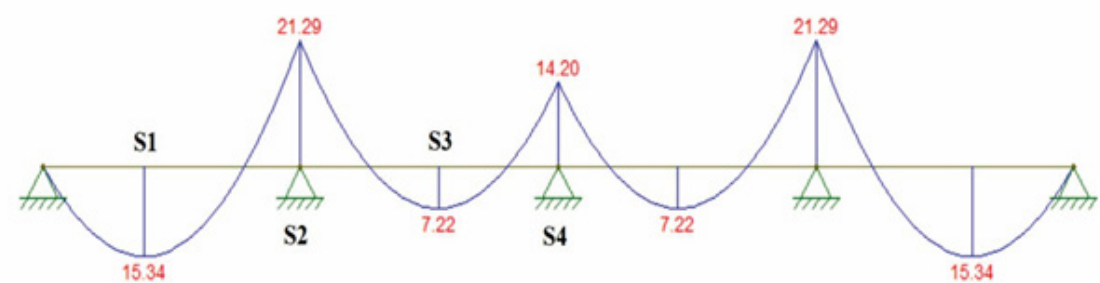

c)

Fonte: Autores. 
Figura 4: Gráficos dos esforços solicitantes das vigas baldrame V04, V05, V06, V07 e V08: a) carga de projeto; b) diagrama de força cortante; c) diagrama de momento fletor.

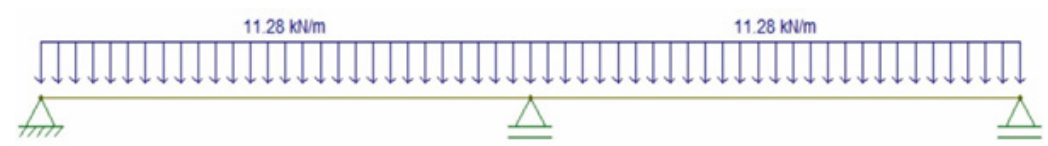

a)

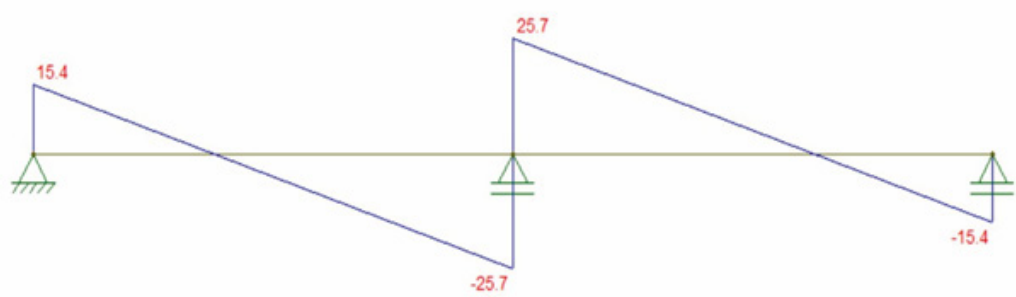

b)

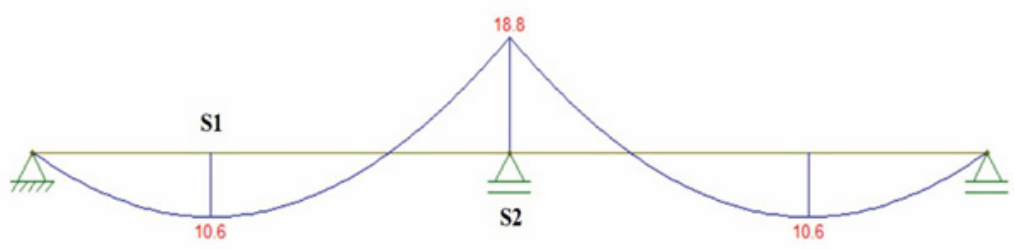

c)

Fonte: Autores

Para o dimensionamento adotou-se o concreto com $\mathrm{f}_{\mathrm{ck}}$ igual a $25 \mathrm{MPa}$, aço CA-50, a classe de agressividade II, para um cobrimento nominal de $3 \mathrm{~cm}$ para vigas ou pilares em contato com o solo, de acordo com ABNT NBR 6118:2014.

A Tabela 3 discrimina a armadura longitudinal ao logo das vigas baldrame, calculadas para o momento máximo e mínimo, divididas por seções (S1, S2, S3 e S4).

Tabela 3: Armadura longitudinal ao longo das vigas, com apoios fixos.

\begin{tabular}{c|c|c|c|c|c}
\hline \multirow{2}{*}{ Viga } & s1 & \multicolumn{2}{|c|}{ s2 } & $s 3$ & $s 4$ \\
\cline { 2 - 6 } & $\mathrm{A}_{\mathrm{s}}\left(\mathrm{cm}^{2}\right)$ & $\mathrm{A}_{\mathrm{s}}\left(\mathrm{cm}^{2}\right)$ & $\mathrm{A}_{\mathrm{s}^{\prime}}\left(\mathrm{cm}^{2}\right)$ & $\mathrm{A}_{\mathrm{s}}\left(\mathrm{cm}^{2}\right)$ & $\mathrm{A}_{\mathrm{s}}\left(\mathrm{cm}^{2}\right)$ \\
\hline $\mathrm{V} 01(15 \times 35)$ & 1,18 & 1,66 & - & 0,81 & 1,09 \\
\hline $\operatorname{V04}(15 \times 30)$ & 0,97 & 1,78 & - & - & - \\
\hline
\end{tabular}

Fonte: Autores.

Nota 1: Pela simetria $\mathrm{V} 01=\mathrm{V} 02=\mathrm{V} 03$ e $\mathrm{V} 04=\mathrm{V} 05=\mathrm{V} 06=\mathrm{V} 07=\mathrm{V} 08$.

Nota 2: $A_{s}$ é a armadura positiva, enquanto $A_{s^{\prime}}$ é a armadura negativa.

A Tabela 4 apresenta o cálculo da área mínima das armaduras para as vigas, de acordo com a ABNT NBR 6118:2014. 
Tabela 4: Armaduras mínimas de flexão para as vigas.

\begin{tabular}{cc}
\hline Viga & $\boldsymbol{A}_{\text {s,min }}\left(\mathbf{c m}^{2}\right)$ \\
\hline $\mathrm{V} 01=\mathrm{V} 02=\mathrm{V} 03$ (seção $15 \times 35 \mathrm{~cm})$ & 0,81 \\
\hline $\mathrm{V} 04=\mathrm{V} 05=\mathrm{V06}=\mathrm{V} 07=\mathrm{V} 08($ seção $15 \times 30 \mathrm{~cm})$ & 0,69 \\
\hline
\end{tabular}

Fonte: Autores.

\section{ANÁLISE ASSUMINDO APOIOS ELÁSTICOS}

Nesta seção é realizado o dimensionamento das vigas baldrame presumido os apoios como elásticos. Admite-se o intervalo entre 20000 até $100000 \mathrm{KN} / \mathrm{m}$ para a rigidez das molas discretas independentes de translação (K), a qual simula a rigidez, respectivamente, de uma argila mole até um silte compactado com areia e muita pedra, segundo Antoniazzi (2011). As análises são concretizadas variando $\mathrm{K}$ em 20000, 40000, 60000, 80000 e 100000 KN/m. Para cada valor de K são determinados seus esforços solicitantes e taxa de armadura à flexão.

A Figura 5 apresenta o esquema estático, enquanto que as Figuras 6 e 7 ilustram os diagramas de força cortante e momento fletor, respectivamente, da viga V01.

Figura 5: Esquema estático da viga V01.

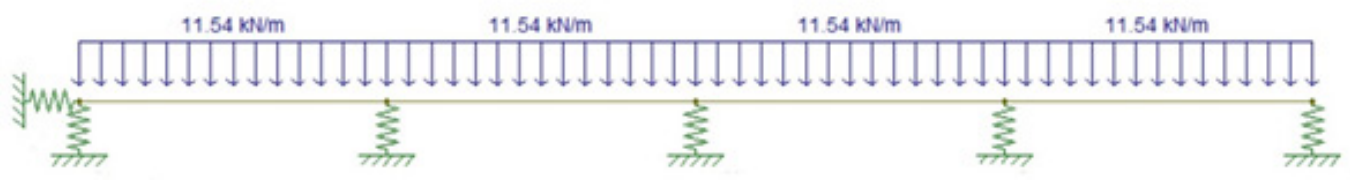

Fonte: Autores.

Figura 6: Diagrama de força cortante da viga V01.

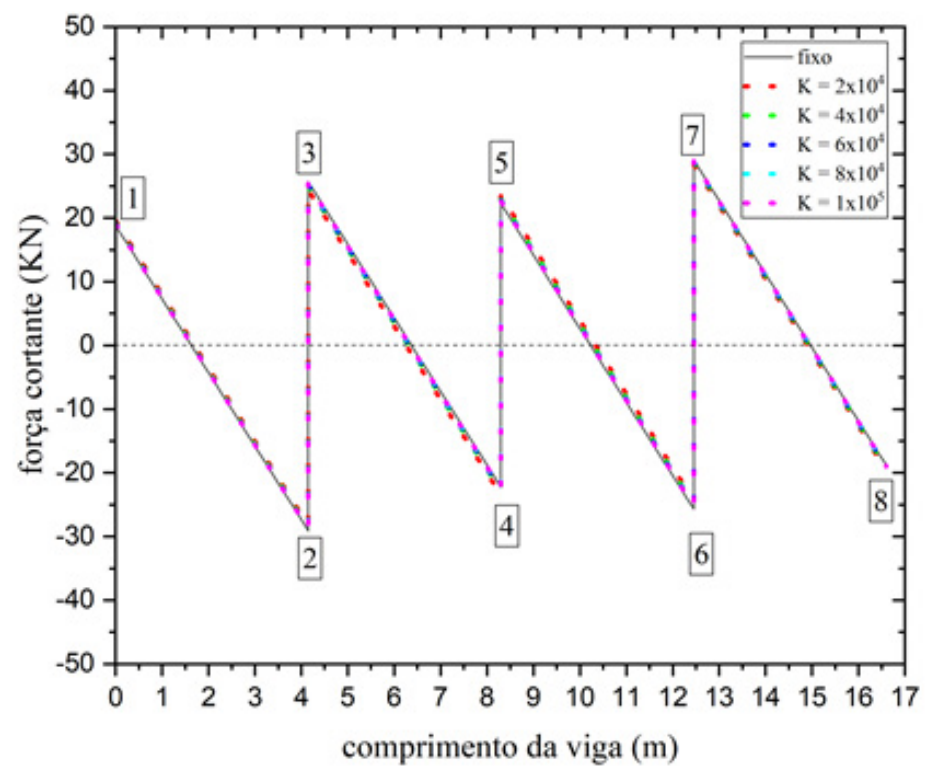

Fonte: Autores.

\section{INTER/SCIENTIA}

REVISTA INTERSCIENTIA | V. 7 | N. 1 | P. 27-39 | JAN-JUN/2019 
Figura 7: Diagrama de momento fletor da viga V01.

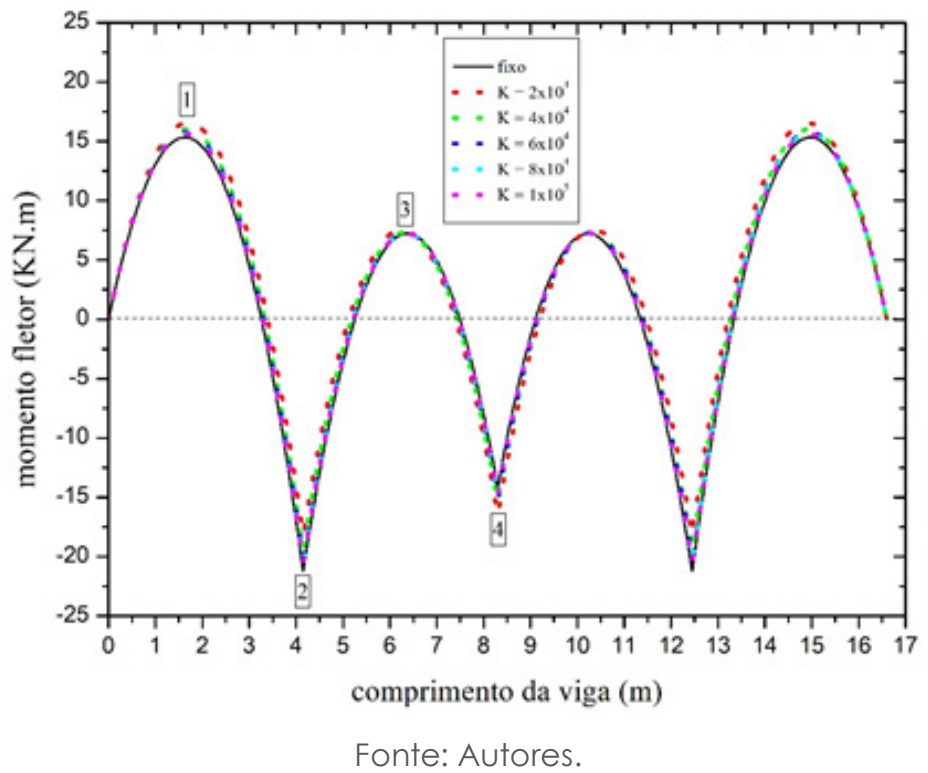

Nota: Convenção de sinal - momento fletor positivo traciona as fibras inferiores da seção transversal.

A Tabela 5 resume a variação da força cortante nos pontos de máximos (1, 3,5 e 7) e mínimos $(2,4,6$ e 8) ao longo comprimento da viga V01.

Tabela 5: Variação da força cortante para cada rigidez: viga V01.

\begin{tabular}{ccccccc}
\hline ponto & $K=\infty$ & $K=2 \times 10^{4}$ & $K=\mathbf{4 \times 1 0 ^ { 4 }}$ & $K=6 \times 10^{4}$ & $K=8 \times 10^{4}$ & $K=1 \times 10^{5}$ \\
\hline 1 & $18,81 \mathrm{KN}$ & $4,0 \%$ & $2,2 \%$ & $1,5 \%$ & $1,2 \%$ & $1,0 \%$ \\
\hline 2 & $-29,08 \mathrm{KN}$ & $-2,6 \%$ & $-1,4 \%$ & $-1,0 \%$ & $-0,8 \%$ & $-0,6 \%$ \\
\hline 3 & $25,66 \mathrm{KN}$ & $-5,1 \%$ & $-2,9 \%$ & $-2,0 \%$ & $-1,6 \%$ & $-1,3 \%$ \\
\hline 4 & $-22,24 \mathrm{KN}$ & $5,8 \%$ & $3,3 \%$ & $2,3 \%$ & $1,8 \%$ & $1,4 \%$ \\
\hline 5 & $22,24 \mathrm{KN}$ & $5,8 \%$ & $3,3 \%$ & $2,3 \%$ & $1,8 \%$ & $1,4 \%$ \\
\hline 6 & $-25,66 \mathrm{KN}$ & $-5,1 \%$ & $-2,9 \%$ & $-2,0 \%$ & $-1,6 \%$ & $-1,3 \%$ \\
\hline 7 & $29,08 \mathrm{KN}$ & $-2,6 \%$ & $-1,4 \%$ & $-1,0 \%$ & $-0,8 \%$ & $-0,6 \%$ \\
\hline 8 & $-18,81 \mathrm{KN}$ & $4,0 \%$ & $2,2 \%$ & $1,5 \%$ & $1,2 \%$ & $1,0 \%$ \\
\hline
\end{tabular}

Fonte: Autores.

Na coluna 2 da Tabela 5 são apresentados os valores de força cortante nos pontos de máximos e mínimos adotando os apoios fixos. Note que nos pontos 1, 4, 5 e 8 houve um aumento desse esforço à medida que $K$ foi diminuído em comparação ao valor da força cortante com apoios fixos. Por outro lado, nos pontos 2, 3, 6 e 7 ocorreu o contrário. Observe que o apoio central (pontos 4 e 5) sofre a maior variação em sua força cortante.

A Tabela 6 sintetiza a variação do momento fletor nos pontos 1, 2, 3 e 4 ao longo comprimento da viga V01. Na coluna 2 são expostos os valores de momento fletor para a viga V01 sobre apoios fixos. Torna-se evidente que ao passo que $\mathrm{K}$ diminui conduz uma maior variação no momento fletor dessa viga. 
Tabela 6: Variação do momento fletor para cada rigidez: viga V01.

\begin{tabular}{ccccccc}
\hline ponto & $\boldsymbol{K}=\infty$ & $\boldsymbol{K}=\mathbf{2 \times 1 0 ^ { 4 }}$ & $\boldsymbol{K}=\mathbf{4 \times 1 0 ^ { 4 }}$ & $\boldsymbol{K}=\mathbf{6 \times 1 0 ^ { 4 }}$ & $\boldsymbol{K}=\mathbf{8 \times 1 0 ^ { 4 }}$ & $\boldsymbol{K}=1 \times 10^{5}$ \\
\hline 1 & $15,34 \mathrm{KN} \cdot \mathrm{m}$ & $7,6 \%$ & $4,4 \%$ & $3,0 \%$ & $2,3 \%$ & $1,8 \%$ \\
\hline 2 & $-21,20 \mathrm{KN} \cdot \mathrm{m}$ & $-14,5 \%$ & $-8,0 \%$ & $-5,5 \%$ & $-4,2 \%$ & $-3,4 \%$ \\
\hline 3 & $7,22 \mathrm{KN} \cdot \mathrm{m}$ & $3,9 \%$ & $1,2 \%$ & $0,7 \%$ & $0,4 \%$ & $0,4 \%$ \\
\hline 4 & $-14,20 \mathrm{KN} \cdot \mathrm{m}$ & $16,2 \%$ & $9,5 \%$ & $6,7 \%$ & $5,2 \%$ & $4,2 \%$ \\
\hline \multicolumn{7}{c}{ Fonte: Autores. } \\
Nota: $\mathrm{K}=\infty$ implica apoio fixo.
\end{tabular}

A Figura 8 exibe o esquema estático, enquanto que as Figuras 9 e 10 apresentam os diagramas de força cortante e momento fletor, respectivamente, da viga V04 para diferentes valores de rigidez.

Figura 8: Esquema estático da viga V04.

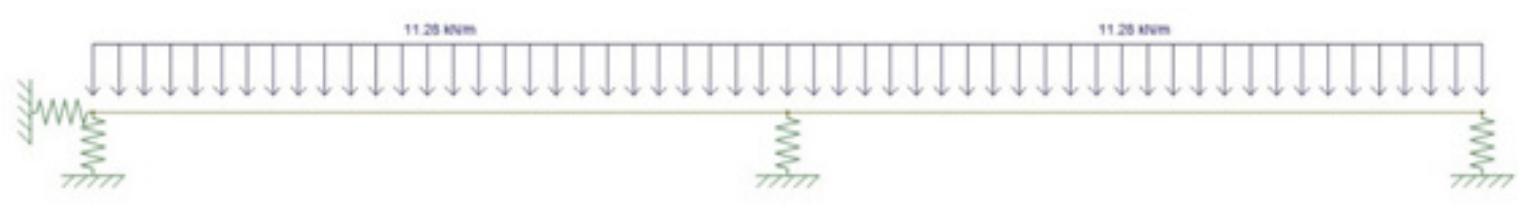

Fonte: Autores.

Figura 9: Diagrama de momento fletor da viga V01.

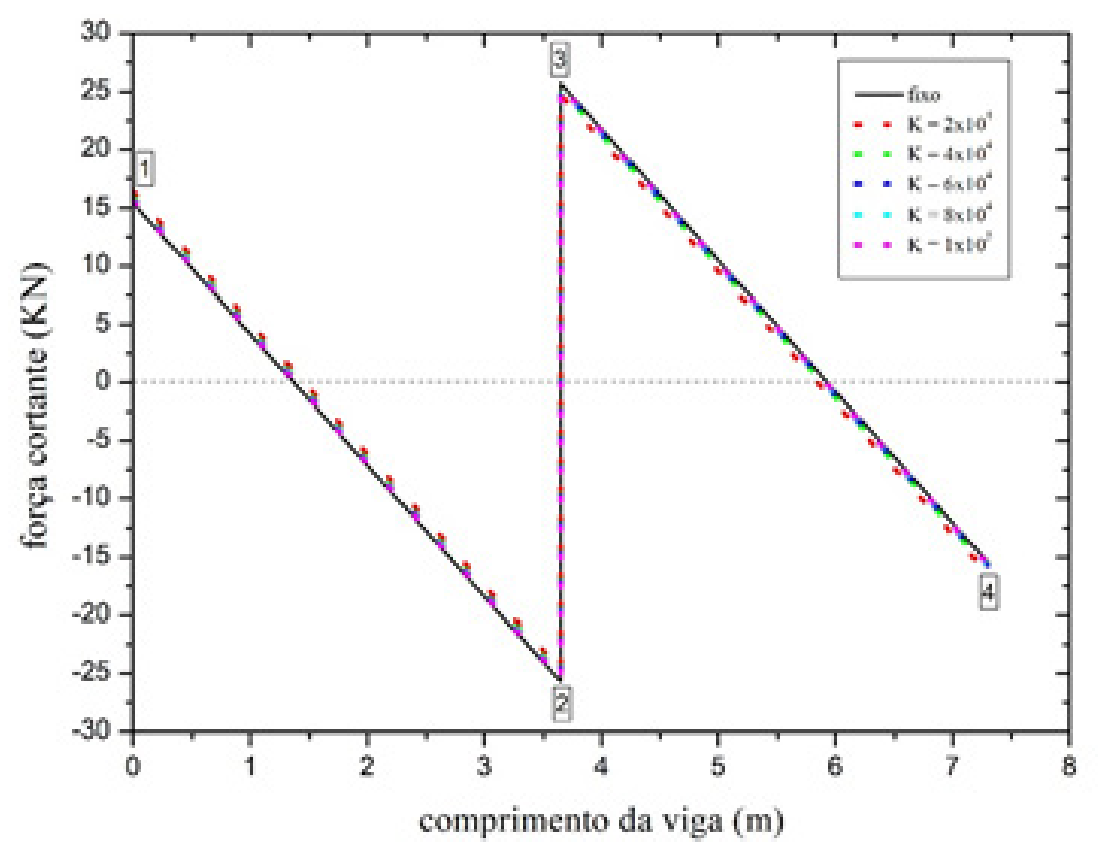

Fonte: Autores. 
Figura 10: Diagrama de momento fletor da viga V04.

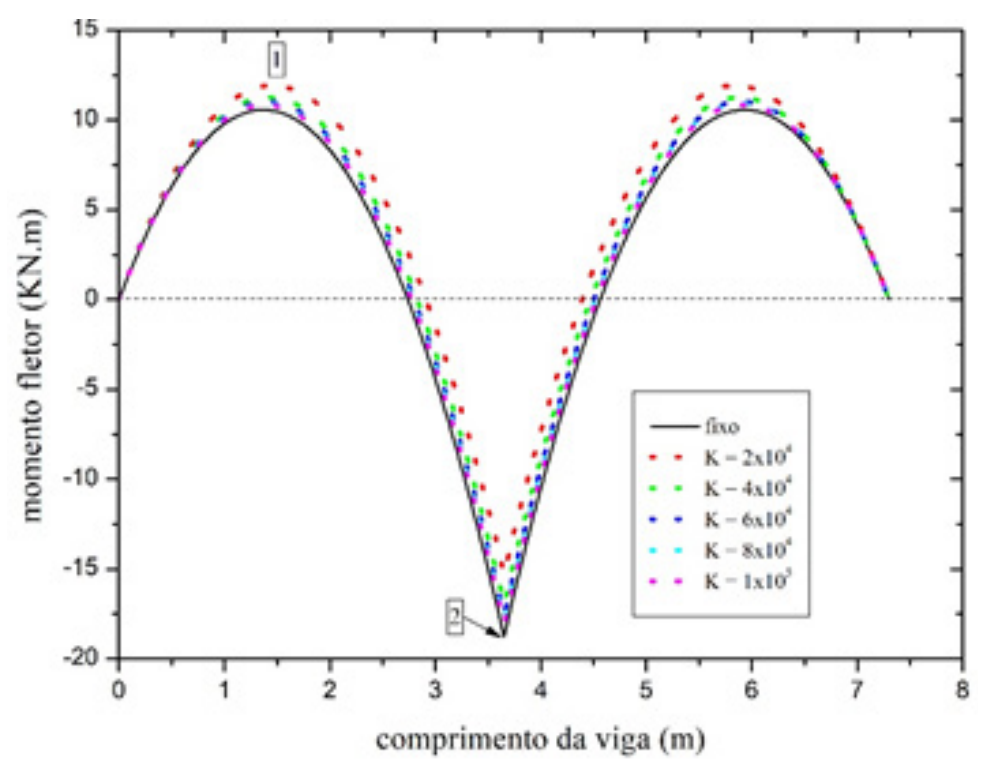

Fonte: Autores.

Nota: Convenção de sinal - momento fletor positivo traciona as fibras inferiores da seção transversal.

Na coluna 2 da Tabela 7 são exibidos os valores de força cortante nos pontos 1, 2, 3 e 4 para a viga V04 presumido apoios fixos. Observe que os apoios externos sofreram as maiores variações nos valores da força cortante, confrontando com os resultados obtidos via apoios indeslocáveis. Com relação a variação do momento fletor dessa viga, Tabela 8, nota-se que a maior variação ocorreu no apoio interno.

Tabela 7: Variação da força cortante para cada rigidez: viga V04.

\begin{tabular}{ccccccc}
\hline ponto & $K=\infty$ & $K=2 \times 10^{4}$ & $K=4 \times 10^{4}$ & $K=6 \times 10^{4}$ & $K=8 \times 10^{4}$ & $K=1 \times 10^{5}$ \\
\hline 1 & $15,44 \mathrm{KN}$ & $6,3 \%$ & $3,2 \%$ & $2,2 \%$ & $1,7 \%$ & $1,4 \%$ \\
\hline 2 & $-25,73 \mathrm{KN}$ & $-3,7 \%$ & $-1,9 \%$ & $-1,3 \%$ & $-1,0 \%$ & $-0,8 \%$ \\
\hline 3 & $25,73 \mathrm{KN}$ & $-3,7 \%$ & $-1,9 \%$ & $-1,3 \%$ & $-1,0 \%$ & $-0,8 \%$ \\
\hline 4 & $-15,44 \mathrm{KN}$ & $6,3 \%$ & $3,2 \%$ & $2,2 \%$ & $1,7 \%$ & $1,4 \%$ \\
\hline
\end{tabular}

Fonte: Autores.

Nota: $K=\infty$ implica apoio fixo.

Tabela 8: Variação do momento fletor para cada rigidez: viga V04.

\begin{tabular}{ccccccc}
\hline ponto & $K=\infty$ & $K=2 \times 10^{4}$ & $K=4 \times 10^{4}$ & $K=6 \times 10^{4}$ & $K=8 \times 10^{4}$ & $K=1 \times 10^{5}$ \\
\hline 1 & 10,57 KN.m & $12,5 \%$ & $6,5 \%$ & $4,4 \%$ & $3,3 \%$ & $2,6 \%$ \\
\hline 2 & $-18,78$ KN.m & $-18,7 \%$ & $-9,7 \%$ & $-6,6 \%$ & $-5,0 \%$ & $-4,0 \%$ \\
\hline
\end{tabular}

Fonte: Autores.

As Tabelas 9 e 10 apresentam a armadura longitudinal nas seções consideradas para as vigas V01 e V04, respectivamente. Torna-se evidente que a consideração dos apoios elásticos reduziu a taxa de armadura dessas vigas. Na linha 3 da Tabela 9, o valor de As é igual para todos os valores de rigidez, pois no 
dimensionamento dessa seção transversal o momento solicitante implicou na taxa de armadura mínima.

Tabela 9: Armadura longitudinal ao longo da viga V01.

\begin{tabular}{c|c|c|c|c|c|c|c}
\hline \multirow{2}{*}{ ponto } & \multicolumn{7}{c}{$\mathrm{A}_{\mathrm{s}}\left(\mathrm{cm}^{2}\right)$} \\
\cline { 2 - 7 } & $K=\infty$ & $K=2 \times 10^{4}$ & \multicolumn{1}{c}{$K=4 \times 10^{4}$} & \multicolumn{1}{c}{$K=6 \times 10^{4}$} & $K=8 \times 10^{4}$ & \multicolumn{1}{c}{$K=1 \times 10^{5}$} \\
\hline 1 & 1,18 & 1,27 & 1,23 & 1,22 & 1,21 & 1,20 \\
\hline 2 & 1,66 & 1,41 & 1,52 & 1,57 & 1,59 & 1,60 \\
\hline 3 & 0,81 & 0,81 & 0,81 & 0,81 & 0,81 & 0,81 \\
\hline 4 & 1,09 & 1,27 & 1,20 & 1,16 & 1,15 & 1,14 \\
\hline
\end{tabular}

Fonte: Autores.

Tabela 10: Armadura longitudinal ao longo da viga V04.

\begin{tabular}{c|c|c|c|c|c|c|c}
\hline \multirow{2}{*}{ ponto } & \multicolumn{7}{c}{$\mathrm{A}_{\mathrm{s}}\left(\mathrm{cm}^{2}\right)$} \\
\cline { 2 - 7 } & \multicolumn{1}{c}{$K=\infty$} & \multicolumn{1}{c}{$K=2 \times 10^{4}$} & $K=4 \times 10^{4}$ & $K=6 \times 10^{4}$ & $K=8 \times 10^{4}$ & \multicolumn{1}{c}{$K=1 \times 10^{5}$} \\
\hline 1 & 0,97 & 1,10 & 1,03 & 1,01 & 1,00 & 0,99 \\
\hline 2 & 1,78 & 1,42 & 1,56 & 1,65 & 1,68 & 1,70 \\
\hline
\end{tabular}

Fonte: Autores.

\section{CONCLUSÃO}

Neste trabalho uma avaliação comparativa entre vigas baldrame com e sem apoios elásticos foi analisada. As análises com apoios elásticos foram concretizadas utilizando molas discretas independentes entre si com rigidez equivalente de um solo argiloso. Averiguou-se que a introdução dos apoios elásticos conduz a uma redução nas taxas de armadura das vigas analisadas. Verificou-se que ao passo que a rigidez relativa entre o solo e a estrutura foi reduzida implicou em um aumento dos momentos fletores positivo com variação máxima de $7,6 \%$ e 12,5\%, respectivamente, para vigas V01 e V04. Por outro lado, os momentos negativos reduziram à medida que a rigidez relativa foi diminuída com variação máxima de 16,2\% e 18,7\% para as vigas V01 e V04, nesta ordem.

\section{AGRADECIMENTOS}

Comentários valiosos dos revisores anônimos são alegremente reconhecidos.

\section{REFERÊNCIAS}

ANTONIAZZI, J. P. Interação solo-estrutura de edifícios com fundações superficiais. Santa Maria, RS: Universidade Federal de Santa Maria, Centro de Tecnologia, 2011. 
ASSOCIAÇÃO BRASILEIRA DE NORMAS TÉCNICAS. NBR 6118: Projeto de estruturas de concreto - procedimentos. Rio de Janeiro, 2014.

ASSOCIAÇÃO BRASILEIRA DE NORMAS TÉCNICAS. NBR 6120: Cargas para o cálculo de estruturas de edificações. Rio de Janeiro, 1980.

CARVALHO, R. C.; Figueiredo, J. R. F. Cálculo e Detalhamento de Estruturas Usuais de Concreto Armado. EdUFSCar, São Carlos, 2014.

CRESPO, V. A. S. Estudo da sensibilidade de edificações em relação ao solo. Campo dos Goytacazes, RJ: Universidade Estadual do Norte Fluminense Darcy Ribeiro, 2004.

LOURINHO, W. R. N. Análise de interação solo-estrutura aplicado à galerias de concreto armado. Rio de Janeiro: Universidade Federal do Rio de Janeiro, 2014.

MEYERHOF, G. G. Some recent foundation research and its application to design. The Structural Engineering, Londres, 1953.

SANTOS, M. G. C. Análise estrutural dos efeitos dos deslocamentos dos apoios de edifícios de paredes de concreto moldadas no local. São Carlos: USP Departamento de Engenharia de Estruturas, 2016 .

SANTOS, P. V.S. Ações evolutivas em edifício de paredes de concreto e de alvenaria, considerando a interação com o solo. São Carlos: USP Departamento de Engenharia de Estruturas, 2016b.

SCARLAT, A.S. (1993). "Effect of Soil Deformability on Rigidity-Related Aspects of Multistory Buildings Analysis". ACI Structural Journal, v.90, n2, Mar-Apr, pp.156-162.

SOUZA, R. A.; REIS, J. H. C. Interação solo-estrutura para edifícios sobre fundações rasas. Maringá, PR: Universidade Estadual de Maringá, Departamento de Engenharia Civil, 2008.

WINKLER, E. Die lehre von Elastizistat und Festigkeit, Domicius. Prag, 1867.

Recebido em: 06/11/2018.

Aceito em: 21/01/2019. 